\title{
Globe
}

\section{Le port de signes religieux dans les écoles québécoises et françaises. Accomodements (dé)raisonnables ou interdiction (dé)raisonnée?}

\section{The Wearing of Religious Symbols in Québécois and French Schools: (Un)Reasonable Accomodations or (Un)Reasoned Ban?}

\section{David Koussens}

Volume 11, numéro 1, 2008

La religion au Québec. Regards croisés sur une intrigue moderne

URI : https://id.erudit.org/iderudit/1000494ar

DOI : https://doi.org/10.7202/1000494ar

Aller au sommaire du numéro

Éditeur(s)

Globe, Revue internationale d'études québécoises

ISSN

1481-5869 (imprimé)

1923-8231 (numérique)

Découvrir la revue

Citer cet article

Koussens, D. (2008). Le port de signes religieux dans les écoles québécoises et françaises. Accomodements (dé)raisonnables ou interdiction (dé)raisonnée? Globe, 11(1), 115-131. https://doi.org/10.7202/1000494ar
Résumé de l'article

Les débats portant sur le port de signes religieux dans les écoles ont trait à la question fondamentale de l'intégration des religions minoritaires dans l'espace public et soulèvent la question de la mise en oeuvre de la neutralité par l'État dans la sphère publique. Le port de signes religieux dans les écoles suscite des débats pour la première fois en France en 1989 et au Québec, en 1994. Sur des fondements différents, mais tout en refusant d'interpréter le signe religieux, ces deux sociétés adoptent alors des positions similaires en autorisant notamment le port du hidjab dans les écoles publiques. Cette situation n’est plus d'actualité. Refusant la visibilité de la diversité religieuse dans l'enceinte de l'institution républicaine qu'est l'école et réaffirmant le rôle de cette institution comme lieu de transmission de valeurs partagées par les citoyens, la France a explicitement interprété le signe religieux pour l'interdire dans les écoles publiques par une loi édictée le 15 mars 2004. Ce faisant, elle confirme son attachement à un modèle d'intégration républicain et s'éloigne ainsi du Canada, où la Cour suprême, en imposant désormais des accommodements raisonnables aux institutions scolaires, promeut l'idée que l'école est un espace de redéfinition des valeurs partagées par les citoyens. 


\title{
LE PORT DE SIGNES \\ RELIGIEUX DANS LES \\ ÉCOLES QUÉBÉCOISES \\ ET FRANÇAISES. \\ ACCOMMODEMENTS \\ (DÉ) RAISONNABLES \\ OU INTERDICTION \\ (DÉ) RAISONNÉE?
}

\author{
DAVID KOUSSENS \\ Universıté du Québec à Montréal
}

Résumé - Les débats portant sur le port de signes religieux dans les écoles ont trait à la question fondamentale de l'intégration des religions minoritaires dans l'espace public et soulèvent la question de la mise en ouvre de la neutralité par l'État dans la sphère publique. Le port de signes religieux dans les écoles suscite des débats pour la première fois en France en 1989 et au Québec, en 1994. Sur des fondements différents, mais tout en refusant d'interpréter le signe religieux, ces deux sociétés adoptent alors des positions similaires en autorisant notamment le port du hidjab dans les écoles publiques. Cette situation n'est plus d'actualité. Refusant la visibilité de la diversité religieuse dans l'enceinte de l'institution républicaine qu'est l'école et réaffirmant le rôle de cette institution comme lieu de cransmission de valeurs partagées par les citoyens, la France a explicitement interprété le signe religieux pour l'interdire dans les écoles publiques par une loi édicrée le 15 mars 2004. Ce faisant, elle confirme son atcachement à un modèle d'intégration républicain et s'éloigne ainsi du Canada, où la Cour suprême, en imposant désormais des accommodements raisonnables aux institutions scolaires, promeut l'idée que l'école est un espace de redéfinition des valeurs partagées par les citoyens.

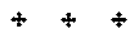




\section{The Wearing of Religious Symbols in Québécois and French Schools: (Un)Reasonable Accomodations or (Un)Reasoned Ban?}

Abstract - The debates surrounding the wearing of religious symbols in school relate to the fundamental question of the integration of religious minorities in public space, and raise the question of the implementation of state neutrality in the public sphere. In 1989, the wearing of religious symbols in school sparked debate for the first time in France; in 1994, the issue was raised in Québec. On different grounds, both societies refused to interpret the religious symbol, and adopted similar positions in authorizing the wearing of the hijab, notably in public school. That position is no longer current; France came to explicitly interpret the religious symbol so as to ban it from public schools with a March 15, 2004 law. In refusing the visibility of religious diversity within the confines of that republican institution, the school, France reaffirmed the role of that institution as a place for the transmission of the citizenry's shared values. With the passage of the 2004 law, France confirmed its attachment to a republican model of integration, and so moved away from Canada, where the Supreme Court, by mandating reasonable accommodations to scholastic institutions, promoted the idea that the school is a space of the redefinition of citizens' shared values.

Le pluralisme des sociétés occidentales contemporaines se caractérise par une multiplicité des conceptions du bien et un accroissement des demandes de reconnaissance des identités fondées sur ces conceptions. Des modèles institutionnels ont été construits dans les États de droit pour tenter de résoudre les contradictions qui apparaissent entre la reconnaissance de certains particularismes et l'affirmation de valeurs communes. La laïcité, qui ne doit plus être appréhendée comme une "exception française ${ }^{t}$ ", mais doit plutôt être envisagée comme un idéal régulateur de la gouvernance politique, peut être considérée comme un outil par lequel l'État va concrètement tenter de réguler le pluralisme dans le domaine religieux. Dans ce sens, elle a été conceptualisée par Micheline Milot comme

[l']aménagement (progressif) du politique en vertu duquel la liberté de religion et la liberté de conscience se trouvent, conformément à une volonté d'égale justice pour tous, garanties par un État neutre à

$$
++
$$

\footnotetext{
1. Comme le souligne Jean Baubérot, l'ıdée que la laıcité seraıt une "exception françaisen, expressıon d'alleurs absente du vocabulaire des pères fondaceurs de la larcité françarse, est apparue dans les années 1990 après les affaires dites " du foulard* (Jean BAUbéroT, Latcté 1905-2005. Entre passon et ratson, Paris, Éditions du Seuil, 2004, p. 177) Cette perception de la laicité comme une spécificité purement hexagonale est historiquement et théoriquement erronée. Ce qui est premier sur le plan historique n'est pas forcément un dérerminant théorıque. En outre, Jean Baubérot soulıgne que des formes de laicité ont pu apparaître, avant même l'adoption de la loi française de 1905, dans de nombreuses autres sociétés (Jean BAUBÉROT, Les latctés dans le monde, Paris, Presses universitares de France, 2007, p. 3).
} 
l'égard des différentes conceptions de la vie bonne qui coexistent dans la société2.

La laïcité comporte des dimensions différentes selon le modèle politique dans lequel elle s'inscrit, que ce modèle soit plutôt libéral ou communautarien. Et quel que soit ce modèle, elle

doit relever les défis qui prennent racine dans les principes mêmes qui la fondent: la neutralité politique et l'égalité de traitement de toutes les expressions sociales de la diversité religieuse, dans les limites du respect des droits d'autrui et de l'ordre public ${ }^{3}$.

Le principe de neutralité de l'État, qui implique la séparation des Églises et de l'État, est ainsi un principe fondateur de la laïcité. Il est aussi un indicateur de la "réalité politique" de laïcités confrontées aux mutations des revendications dans les États modernes et il permet d'évaluer ces différentes laïcités les unes par rapport aux autres en prenant en compte notamment les fondements philosophiques qui les ont légitimées et leur réalité sociale actuelle $e^{4}$. En effet, la séparation peut être mise en œuvre différemment selon les États et sa formalisation dans une norme juridique ne doit donc pas être considérée comme une garantie de son effectivité. Si "la séparation des pouvoirs demeure un principe important, en tant que "condition" de mise en œuvre de la neutralité", elle peut aussi s'insérer "dans l'action politique comme une composante effective de la mise en œuvre de la neutralité par l'État $"$. La première conception est bien illustrée par la France, où la séparation, formalisée par la loi de 1905 dissociant les Églises et l'État, a été indispensable pour envisager une mise à bas des prétentions terrestres de l'Église catholique. Sous certains aspects, elle perdure dans la mise en ceuvre française de la neutralité de l'État. La seconde conception, quant à elle, se retrouve plutôt dans des pays comme le Canada, où la séparation n'est pas inscrite dans une norme juridique formelle et où le principe de neutralité de l'État n'est énoncé dans aucun texte constitutionnel. À l'inverse même, puisque la Constitution énonce que "le Canada est fondé sur des principes qui reconnaissent la suprématie de Dieu». La neutralité reste pour autant bien réelle et ressort du

$$
+4
$$

2. Micheline MILOT, Laicuté dans le nouveau monde. Le cas du Québec, Turnhout, Brepols, 2002, p. 34.

3. Micheline MILOT, "Neutraltté politique er libertés de religion dans les sociétés plurielles. Le cas canadien", Jean

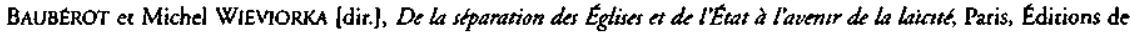
l'Aube, 2005, p. 283.

4. Jean BAUBÉROOT, Les latcités dans le monde, p. 5.

5. Mıcheline MILOT, "Neutralıré polıtique et libertés de religion dans les sociétés plurielles. Le cas canadien a, p. $275-276$. 
traitement équitable des religions et de la protection juridique accordée à la liberté de religion par les Chartes canadienne et québécoise des droits de la personne'.

En France et au Québec, les débats portant sur le port de signes religieux dans les établissements scolaires ont connu de larges développements dans les années 1990. Ce n'est qu'en 2004 et en 2006 que les politiques à appliquer ont été fixées par la loi en France et par la Cour suprême au Canada. Il est utile d'étudier le sort encouru par le signe religieux dans les écoles parce qu'il révèle les modes de déploiement de la laïcité, et particulièrement de la conception de la séparation des Églises et de l'État, dans un modèle institutionnel libéral d'influence républicaine et dans un modèle institutionnel libéral d'influence multiculturaliste. Si le port du foulard islamique en particulier a d'abord été autorisé dans les établissements scolaires français et québécois, les fondements juridiques présidant à cette autorisation sont différents et s'inscrivent déjà dans des principes de philosophie politique propres aux choix politiques de régulation du pluralisme qui ont été faits en France et au Canada.

À partir de 2004, l'éloignement des positions adoptées concernant le port de signes religieux dans les écoles révélera plus explicitement la divergence dans le type de neutralité promu par les États, et ainsi le choix du mode de régulation du religieux applicable. Pourtant, en interdisant le port de signes religieux, la France allait se fonder sur le concept canadien d' "accommodement raisonnable" dont elle allait cependant déformer le sens.

\section{DES MOYENS DIFFÉRENTS POUR UNE FIN SIMILAIRE. LAUTORISATION DU PORT DU FOULARD ISLAMIQUE DANS LES ETABLISSEMENTS D'ENSEIGNEMENT EN FRANCE ET AU QUÉBEC (1989-2003)}

La question du port du foulard islamique dans les écoles a suscité de nombreux débats au Québec et en France dans les années 1990. Si, de part et d'autre de l'océan Atlantique, les jeunes filles ont eu l'autorisation de porter

$$
+4
$$

6. Dans l'arrêt Syndicat Northcrest contre Amselem, la Cour suprême du Canada définir la liberté de religion comme "la liberté de se livrer à des pracıques et d'entretenır des croyances ayant un lıen avec une religion, pratıques et croyances que l'intéressé exerce ou manifeste sincèrement, selon le cas, dans le but de communiquer avec une entı́é divıne ou dans le cadre de sa foi spirituelle, indépendamment de la question de savorr si la pratique ou la croyance est prescrite par un dogme religieux officiel ou conforme à la position des représentants religieux». La Cour suprême précıse alors que "ćest le caractère spirituel ou religieux d'un acte qui encraîne la protection [par les Chartes des droits] "(Syndzcat Northcrest c. Amselem, [2004] 2 R.C.S.551). 
ce signe religieux dans les établissements qu'elles fréquentaient, c'est pourtant pour des motifs différents.

\section{Lautorisation française du port du foulard islamique dans les établissements d'enseignement}

Afin que les libertés de conscience et de religion soient complètes, l'État français ne doit pas se préoccuper des convictions religieuses des citoyens. $\mathrm{La}$ loi de séparation des Églises et de l'État lui impose une obligation négative ${ }^{7}$ dans son article 2: "La République ne reconnaît, ne salarie ni ne subventionne aucun culte». La neutralité de l'État a pour objet de protéger la liberté de conscience et de religion. Pour cette raison, elle ne s'impose pas à tous de la même façon. Si le principe de neutralité est obligatoire pour les agents de

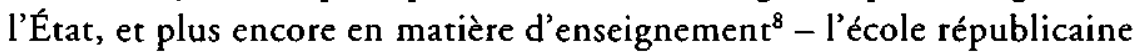
gardant sa vocation de former des esprits libres et éclairés -, les élèves doivent pouvoir manifester leurs libertés de conscience et de religion au sein de l'école qu'ils fréquentent. Le port du foulard islamique par les élèves en France doit être appréhendé au regard de ce paramètre.

Le gouvernement français n'est pas intervenu directement pour gérer la question du port du foulard dans les écoles et c'est le Conseil d'État qui, à partir de 1989, a eu à déterminer si le port de signes religieux à l'école était compatible avec le principe républicain de laïcité. Après avoir été saisi par le ministre de l'Éducation nationale de cette question, le Conseil d'État, dans sa fonction de conseil du gouvernement ${ }^{9}$, a rendu un avis délimitant les conditions dans lesquelles le port du foulard islamique est admis dans les établissements d'enseignement ${ }^{10}$. Les élèves bénéficient de la liberté de conscience et d'expression, et cette liberté "comporte pour eux le droit d'exprimer et de manifester leurs croyances religieuses à l'intérieur des établissements scolaires, dans le respect du pluralisme et de la liberté d'autrui». Ils peuvent donc porter des signes religieux à l'école, mais ce port doit respecter «les activités d'enseignement [et le] contenu des programmes" ainsi que les

$$
+4
$$

7. Genevieve KoUBI, *Vers une evolution des rapports entre ordre juridique et systèmes religieux? ", Jurisclasseur Ptrodique, 1987, p. 3292.

8. CE (Conseil d'Étar), 3 mal 2000, Mademoiselle Marteaux, $n^{\circ} 217017$.

9. Juge suprême en droir administracif, le Conseil d'État est également conseiller du gouvernement. Cette seconde fonction l'amène à se prononcer sur tous les projets de lois et d'ordonnances avant que ceux-ci ne soient transmis au Conseil des ministres. Elle l'amène aussi à être consulté par le gouvernement sur toute question susceptible de poser des difficultés sur le plan juridique. C'est donc à ce titre qu'ıl a écé saisı de la question du port de signes religreux dans les écoles publiques.

10. Avis du Consenl d'Etat portant sur la questron de savour si le port de signes d'appartenance d̀ une communauté religieuse est ou non companble avec le principe de laicité, Assemblée générale (seccion de l'incérneur), 27 novembre 1989, $n^{\circ} 346893$ (pour cette ciration et les suivates). 
objectifs plus généraux de l'enseignement républicain: "l'acquisition par l'enfant d'une culture et sa préparation à la vie professionnelle et ses responsabilités d'homme et de citoyen [...], le développement de sa personnalité [...], l'égalité entre les hommes et les femmes". Le Conseil d'État affirme alors clairement:

Le port par les élèves de signes par lesquels ils entendent manifester leur appartenance à une religion n'est pas par lui-même incompatible avec le principe de laïcité dans la mesure où il constitue l'exercice de la liberté d'expression et de manifestation de croyances religieuses.

Cependant, cette compatibilité est générale. Les juges du PalaisRoyal énoncent alors, dans une formule devenue célèbre, les mesures à y apporter. Les libertés d'expression et de religion doivent être conciliées avec le droit à la santé, la liberté de l'enseignement et des principes d'ordre public: la dignité de la personne humaine et la sécurité publique. C'est à l'autorité dans l'établissement d'enseignement que revient d'apprécier, au cas par cas, si les conditions dans lesquelles une jeune femme porte le voile dans son établissement sont compatibles avec le principe de laïcité. Le juge vérifie des situations concrètes, mais ne peut en aucun cas se prononcer sur le signe en tant que tel ${ }^{11}$. Ainsi, il est interdit pour un proviseur, sauf circonstances particulières, d'édicter un règlement refusant l'admission à l'école d'étudiants "la tête couverte", car une telle interdiction, par sa permanence, porte atteinte à la liberté d'expression des élèves ${ }^{12}$.

En tant qu'institution de l'État, le Conseil d'État est astreint au principe de neutralité. Il ne peut donc se prononcer sur la signification à attribuer à un signe religieux ${ }^{13}$. Le danger de l'interprétation serait très grand, car la signification attribuée au port du foulard par les jeunes filles ellesmêmes n'est pas univoque et peut recouvrir un ensemble de symboliques très diverses. C'est pour cette raison que le signe religieux ne pouvait être considéré, par lui-même, comme incompatible avec la laïcité. Pour l'interdire, le juge aurait dû «qualifier le signe au regard de sa qualification religieuse, dégager sa portée pour la foi musulmane et en déduire son incompatibilité avec les principes que l'école doit véhiculer ${ }^{14}$ ". Autrement

$$
++4
$$

11. CE (Conseil d'État), 2 novembre 1992, Monsieur Kherouaa et autres, Recueil Lebon, p. 389.

12. CE (Consell d'Érat), 14 mars 1994, Mesdemorselles $\mathrm{N}$ er $\mathrm{Z}$. Yilmaz, $\mathrm{n}^{\circ} 145656$

13. David KESSLER, «Laicité. Du combat au drot [entretıen avec Marcel Gauchet] „, Les Debats, $\mathrm{n}^{\circ} 77$, novembredécembre 1993, p. 99.

14. David KESSLER, «Prudence de l'ıncérêr général”, La prudence, une morale du possible, Édıtions Autrement, $n^{\circ} 20$, octobre 1996, p. 113. 
dit, interpréter le signe, cela aurait été affirmer que les valeurs défendues par l'islam, et auxquelles adhèrent une grande communauté immigrante en France, étaient incompatibles avec le principe de la laïcité et les valeurs de la République. Certaines expressions sociales de la foi peuvent donc sembler particulièrement choquantes au regard des valeurs publiques, mais elles sont protégées par le principe de la liberté religieuse dans le cadre des limites qui lui sont imparties. Ainsi, pendant 14 ans, le port de signes religieux, en général, et du foulard islamique, en particulier, est officiellement autorisé dans les établissements d'enseignement public.

\section{L'accommodement québécois au port du foulard islamique dans les établissements d'enseignement}

Au Québec, la Commission des droits de la personne et des droits de la jeunesse $^{15}$ a adopté et rendu public le 21 décembre 1994 un avis sur le port du foulard islamique dans les écoles ${ }^{16}$. Si des conclusions analogues à celles du Conseil d'État français en ressortent, elles ne sont pas fondées sur le même raisonnement juridique. Selon Pierre Bosset, alors directeur de la recherche et de la planification de la Commission:

la problématique des rapports entre l'État et les religions évolue [...] au Canada et au Québec dans un cadre différent de celui de la France. Le fondement principal de ce cadre est la garantie constitutionnelle des libertés fondamentales de conscience et de religion et du droit à l'égalite $e^{17}$.

Ce droit à l'égalité doit ici être compris comme un droit à une égalité de fait, une égalité concrète de tous les citoyens dans l'espace public. Et le droit canadien va tâcher de corriger toute éventuelle discrimination indirecte, celle-ci étant appréhendée comme celle qui «découle d'une règle "neutre" c'est-à-dire qui s'applique de la même façon à tous, mais qui produit néanmoins un effet discriminatoire sur un seul groupe de personnes en ce

$$
++
$$

15. La Commission québécoise des droits de la personne et des droits de la jeunesse dispose, d'une part, du mandar de promouvoir et d'éduquer aux droits fondamencaux ainsi que de conseller le gouvernement dans ces domaines, d'aurre part, d'un mandat consistant à réaliser des enquêtes sur les plantes qui luı sont adressées pour discrimınation. Suite à ces enquêtes, la Commission émet des recommandacions puis saisit le Tribunal des droits de la personne si ces recommandations ne sont pas suivies.

16. COMMISSION DES DROITS DE LA PERSONNE ET DES DROITS DE LA JEUNESSE, "Le port du foulard islamique dans les écoles publiques $n$, avis du 21 decembre 1994 (COM-388-6.1.1).

17. Pierre BOSSET, Le foulard islamique et l'egalité des sexes. Reflexcons sur le discours juridique instisutionnel en France et au Québec, texte consulté sur le sire "Les Éditions Thémis", http://www.themis.umontreal.ca/consultation_gratuicel droirs_fondamentaux/15_bosser.pdf (15 février 2007). 
qu'elle leur impose des obligations ou des conditions restricrives ${ }^{18}$ ". Pour limiter toute atteinte au principe d'égalité, la Cour suprême du Canada a défini en 1985 un corollaire du principe d'égalité. Il s'agit du principe de l'accommodement raisonnable ${ }^{19}$ obligeant

dans certains cas l'État, les personnes ou entreprises privées à modifier des normes, des pratiques ou des politiques légitimes et justifiées, qui s'appliquent à tous sans distinction, pour tenir compte des besoins particuliers de certaines minorités, surtout ethniques et religieuses ${ }^{20}$.

Cette obligation dégagée par la Cour suprême dans le cadre d'un conflit de droit privé, en l'occurrence de droit social, ne se limite pas pour autant à cette sphère. Toutes les institutions publiques y sont assujetties. L'obligation d'accommodement ne revient pas à la victime de la discrimination, mais à l'entreprise ou à l'institution qui adopte une norme susceptible de créer une situation discriminatoire. La personne concernée a essentiellement pour obligation de présenter la façon dont la norme contestée porte préjudice à son droit à l'égalité, puis de collaborer avec l'institution en lui faisant connaître ses besoins et en acceptant une solution raisonnable. Cette solution pourra alors consister à dispenser l'intéressé de l'application de la règle engendrant une discrimination ou à mettre à sa disposition certaines installations particulières ${ }^{21}$. L'accommodement raisonnable trouve toutefois plusieurs limites: «le caractère raisonnable de la norme ou de la règle, l'effort déployé pour trouver un accommodement, le caractère excessif de la contrainte imposée à l'employeur ou à l'institution [...] et l'atteinte au droit d'autrui ${ }^{22}$ ».

L'accommodement raisonnable est donc un principe jurisprudentiel qui est examiné au cas par $\mathrm{cas}^{23}$. Comme le relève Micheline Milot, «il s'agit d'un dispositif de nature progressive et additive, qui est avant toute chose une mesure "pragmatique" et non pas théorique ${ }^{24}$ ". Cette mesure

$$
++4
$$

18. José WOEHRLING, „L'obligatıon d'accommodement raisonnable et l'adaptation de la socıété à la diversité religieuse”, Revue de drott de McGtlh, vol. 43, 1998, p. 331-332.

19. « L'obligation dans le cas de la discrimination par suite d'un effect préjudiciable, fondée sur la religıon ou la croyance, conssste à prendre des mesures raisonnables pour s'entendre avec le plaignant, à moins que cela ne cause une contrainte excessive [.. ] il s'agit de prendre les mesures qui peuvent être raisonnables pour s'entendre sans que cela n'entrave indument l'exploitation de l'entreprise de l'employeur et ne lui impose des fras excessifsn (O'Malley c Simpson-Sears, [1985] 2.R.C.S.536).

20. José WOEHRLING, op. cat., p. 328.

21. COMMISSION DES DROITS DE LA PERSONNE ET DES DROITS DE LA JEUNESSE, "Centre de recherche-action sur les relations raciales v/s École de technologıe supérieure ex R.N*, Résolution COM-510-5.2.1, 3 février 2006.

22. Micheline MILOT, Latcite dans le nouveau monde. Le cas du Quebec, p. 129.

23. Central Okanagan School Dustrict No.23 c Renaud [1992] 2 R.C.S.970.

24. Micheline MiLOT, Laicuté dans le nouveau monde. Le cas du Québec, p. 128. 
pragmatique peut découler d'accords amiables entre les parties ou, si aucun terrain d'entente n'est trouvé, être recommandée par la Commission des droits de la personne ou ordonnée par un tribunal.

La Commission des droits de la personne et des droits de la jeunesse, dans l'avis rendu le 21 décembre $1994^{25}$, va donc s'appuyer sur les dispositions de la Charte québécoise des droits et libertés ainsi que sur le principe jurisprudentiel en droit canadien de l'accommodement raisonnable. Ce dernier principe doit s'appliquer en matière d'éducation, car il est "une condition indispensable à l'exercice en pleine égalité du droit à l'instruction publique ${ }^{26} »$. La Commission rappelle que les étudiants disposent du choix de l'établissement scolaire où ils veulent étudier. Dans ce cadre, une mesure fondée même indirectement sur la religion, telle l'interdiction du port de vêtements religieux, qui empêcherait lesdits étudiants de fréquenter telle ou telle école, et de fait les marginaliserait, constitue alors une discrimination au regard du droit à l'instruction publique. La Charte est donc violée. Dès lors, l'avis affirme que les établissements scolaires ont l'obligation d'adapter leur règlement interne aux situations individuelles des étudiantes portant le foulard pour autant que certains "éléments essentiels" du système scolaire public soient préservés. C'est ce qui permettra de juger du caractère raisonnable ou excessif de l'accommodement demandé. La Commission relève ainsi que les accommodements doivent respecter la nature de l'institution scolaire québécoise, ce qui implique:

le respect des programmes officiels d'enseignement, le statut de la langue française en tant que langue d'enseignement de même que l'égalité des sexes [auxquels s'] ajoutent nécessairement, des considérations relatives au maintien de l'ordre, à la sécurité et à l'efficacité pédagogique. Tous ces critères sont des éléments de l'ordre public, des valeurs démocratiques et du bien-être général, intérêts dont peuvent découler certaines limites à l'exercice des libertés fondamentales ${ }^{27}$.

Dans des termes proches de ceux employés par le Conseil d'État, la Commission précise en outre que «le port du foulard islamique doit en principe être considéré licite, sauf s'il s'inscrit dans un contexte de pression

$$
+4
$$

25. COMMISSION DES DROITS DE LA PERSONNE ET DES DROITS DE LA JEUNESSE, "Le port du foulard islamique dans les écoles publiques*, avis du 21 décembre 1994 (COM-388-6.1.1).

26. COMMISSION DES DROITS DE LA PERSONNE ET DES DROTTS DE LA JEUNESSE, “Le pluralısme religieux au Québec. Un défi d'éthıque socialen, document soumis à la réflexion publique, février 1995, Cat. 7.113-2.1.1, p. 23.

27. Ibed 
sur les élèves, de provocation ou d'incitation à la discrimination fondée sur le $\operatorname{sex}^{28}$ ". Le principe de l'égalité des sexes est l'un des droits que les enseignants ont l'obligation de promouvoir à l'école. Par conséquent, il a fallu que la Commission envisage le foulard en tant que signe d'une expression religieuse et se détache des diverses interprétations qu'il recouvre pour en autoriser le port dans les écoles. Dans son avis rendu le 21 décembre 1994, elle précise qu’à moins que la preuve contraire n'ait été apportée, le foulard, qui traduit l'expression d'une conviction religieuse, ne peut être considéré comme reflétant une aliénation de la volonté de l'élève. Une telle position ferait «injure" à l'expression de l'étudiante. Jusqu'en 2003, les solutions adoptées par la France et le Québec sont ainsi très similaires. Elles se fondent cependant sur deux raisonnements différents. En France en 1989, le port du foulard islamique est autorisé, au nom de la liberté de conscience et de la liberté d'expression, pourvu que ce port ne soit pas susceptible de troubler l'ordre public et qu'il respecte les principes véhiculés par l'institution républicaine qu'est l'école. Au Québec, afin de préserver les libertés de conscience er de religion, le droit à l'égalité et celui à l'instruction publique, c'est l'école qui a l'obligation d'accommoder son règlement à la situation de jeunes filles portant le voile pourvu que cet accommodement n'entraîne pas de contrainte excessive pour l'institution et que le port du voile respecte certaines prescriptions d'ordre public.

\section{DES MOYENS FAUSSEMENT SIMILAIRES POUR UNE FIN DIFFERENTE.}

\section{QUELS ACCOMMODEMENTS AU PORT DE SIGNES RELIGIEUX DANS LES ÉTABLISSEMENTS D'ENSEIGNEMENT FRANÇAIS ET CANADIENS ? (2004-2006)}

La question du port de signes religieux dans les établissements d'enseignement va prendre un tour nouveau à l'aube du XXI ${ }^{e}$ siècle. Le débat est toujours ouvert et les solutions apportées, en France et au Québec, divergent. Traumatisée par l'élection présidentielle de mai 2002, aboutissement d'une fracture sociale toujours plus profonde, la société française semble se remettre en question. La recherche d'une nouvelle cohésion sociale s'impose. Dans une allocution du 3 juillet 2003, le président de la République française indique vouloir rouvrir le débat de la laïcité. Il confie à Bernard Stasi la présidence d'un groupe de réflexion afin de mettre en œuvre «une laïcité

$$
++4
$$

28. Ibud. p. 23 et 26 . 
garante de la cohésion nationale et du respect des différences de chacun ${ }^{29}$ ". $\mathrm{Au}$ Québec, le système scolaire a subi des transformations majeures sur le plan de la confessionnalité ${ }^{30}$ et ces transformations ont alors "fait surgir de façon plus explicite [...] la diversité religieuse des élèves dans les écoles publiques, en y ajoutant la mixité entre catholiques et protestants ${ }^{31}$ ". La visibilité accrue de la diversité religieuse à l'école soulève de nouvelles questions sur le plan de l'accommodement raisonnable.

\section{Quand la fin justifie les moyens.}

\section{La déformation française}

\section{du principe canadien d'accommodement raisonnable}

Le rapport présenté par la Commission de réflexion sur l'application du principe de laïcité dans la République (rapport Stasi) indique que l'école doit permettre de construire "un destin commun " à tous les Françaiis ${ }^{32}$. Affirmant que la laïcité de combat, nécessaire à l'établissement de bases stables à la République, n'est plus d'actualité, il souligne que la diversité religieuse à l'école est une richesse. Mais il considère que ce projet est actuellement confronté au communautarisme croissant et que ce communautarisme est porteur d'exclusion. Le rapport Stasi rappelle que «le principe de neutralité est la première condition de la laïcités ${ }^{33}$ ". Il est le corollaire du principe d'égalité des citoyens et implique en ce sens que tous les usagers du service public doivent être traités de la même façon par l'administration. Pour cette raison, "l'administration, soumise au pouvoir politique, [doit donner] non seulement toutes les garanties de la neutralité, [mais aussi en présenter] les apparences pour que l'usager ne puisse douter de sa neutralité». Pour ne pas douter de la neutralité de l'État, il faut que l'usager ne se sente pas discriminé, même indirectement, par une loi «neutre» qui l'empêcherait

$$
+4
$$

29. Jacques CHIRAC, “Lettre de mission a Bernard Stasi», 3 juillet 2003, hrtp://www.elysee.fr/elysee/elysee.fr/ francars_archıves/interventions/letres_et_messages/2003/juiller/lettre_de_mission_du_president_de_la_republique_a_ m_bernard_stasi_president_de_la_commission_de_reflexion_sur_l_application_du_principe_de_laefcıte_dans_la_repu blique. $1037 . h t m l$ (14 seprembre 2007).

30. Amorcée en 1995 er 1996, la réforme de l'éducation au Québec a pris corps avec l'adoprion de deux lois La loi

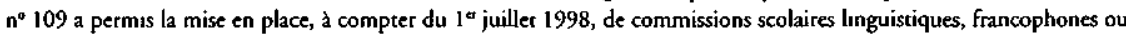

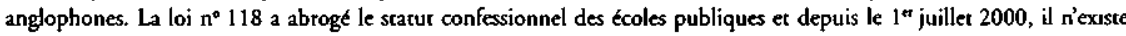
plus au Québec d'école publique reconnue comme catholique ou protestante.

31. COMITE SUR LES AFFAIRES RELIGIEUSES, "Rites et symboles relıgıeux à l'école. Défis éducatifs de la diversité *, Avis au Ministre de l'éducation, mars 2003, hrtp://www.mels.gouv.qc.ca/affairesreligieuses/CAR/PDF

IAbrege_expressions\%20religieuses.pdf (30 mars 2007).

32. COMMISSION DE REFLEXION SUR L'APPLICATION DU PRINCIPE DE LAICITE DANS LA RÉPUBLIQUE, Rapport remis au président de la République le 11 décembre 2003, p. 17, consulté sur le site http://lesrapports.ladocumentation francaise fr/BRP/034000725/0000.pdf (30 mars 2007).

33. Ibid, p. 22. 
d'exercer sa liberté de religion. Pour cette raison, le rapport souligne que "les exigences d'une neutralité absolue sont donc tempérées par les "accommodements raisonnables" permettant à chacun d'exercer sa liberté religieuse $\mathrm{e}^{34}$ \%. De tels accommodements sont déjà consentis par les pouvoirs publics. Ainsi, le rapport relève que des autorisations d'absence au travail dans les services publics peuvent être données lors de jours de fêtes religieuses, que des locaux publics peuvent être prêtés à des organisations communautaires pour célébrer ces fêtes et que les services publics prennent souvent en compte les interdits alimentaires liés aux pratiques religieuses. Par ailleurs, les municipalités autorisent de plus en plus facilement la construction d'édifices cultuels et, dans certains cas, des baux emphytéotiques portant sur des bâtiments publics sont conclus avec des associations cultuelles afin de leur permettre d'y exercer le culte. Le rapport préconise alors certains aménagements et, se référant expressément au concept canadien d'accommodement raisonnable $e^{35}$, demande notamment que "des substituts au porc et le poisson le vendredi [soient] proposés dans le cadre de la restauration collective" sans pour autant que ces mesures n'entravent le fonctionnement normal des services publics.

Cependant, ce que le rapport Stasi qualifie d'accommodement raisonnable ou qu'il recommande ici en tant que tel ne correspond pas tout à fait au concept juridique dégagé par la jurisprudence de la Cour suprême du Canada. Si le rapport envisage effectivement l'accommodement comme une mise à disposition de certaines installations ou l'octroi de certains avantages à des communautés religieuses, il n'évoque pas le fait que des personnes issues de ces communautés puissent obtenir une dispense dans l'application d'une norme de droit qui, de fait, entraverait la libre expression de leurs convictions religieuses. La substance même du principe juridique de l'accommodement raisonnable est donc ici déformée. Mais plus encore qu'une simple déformation, le rapport allait expressément en inverser le sens et cette inversion ne sera pas sans effet sur la recommandation politique visant à interdire le port des signes religieux jugés ostentatoires dans les établissements d'enseignement publics.

Après avoir rappelé que la laïcité implique «l'indépendance du pouvoir politique et des différentes options spirituelles ou religieuses» et

$$
+4
$$

34. Ibid, p. 23

35. Ibrd, p. 64. Le rapport indique ic1 que certe proposition correspond "au principe que les Québécois appellent "l'accommodement raısonnable" ". On notera que ce pruncıpe, dégagé par la Cour suprême du Canada, est plus canadien que québécors. 
donc la neutralité de l'État, le rapport Stasi précise que celui-ci «ne saurait [pour autant] recouvrir d'un voile d'ignorance le fait spirituel ou religieux". Une exigence de protection de la liberté de conscience et, plus précisément, de garantie de la libre expression des croyances s'impose donc à lui. Mais cette exigence découlant du principe de laïcité n'est pas unilatérale. C'est une "exigence partagée", notamment dans le cadre scolaire. Et c'est en évoquant le partage de cette exigence que le rapport Stasi va inverser le sens du concept canadien d'accommodement raisonnable. Le rapport indique en effet ceci:

l'exigence laïque demande [...] à chacun un effort sur soi [...]. Le citoyen conquiert par la laïcité sa liberté de conscience; en contrepartie il doit respecter l'espace public que tous peuvent partager. Revendiquer la neutralité de l'État semble peu conciliable avec l'affichage d'un proselytisme agressif, particulièrement dans l'espace scolaire. Accepter d'adapter l'expression publique de ses particularités confessionnelles et de mettre des bornes à l'affirmation de son identité permet la rencontre de tous dans l'espace public. C'est ce que les Québécois qualifient d'" accommodement raisonnable ${ }^{36}$ ".

La distance est prise avec la position canadienne dans la mesure où, pour ce qui concerne l'école, toute dispense possible dans l'application d'une règle "neutre", mais qui entraverait la libre expression des croyances des élèves, est annihilée par les limites que ceux-ci doivent eux-mêmes s'imposer dans l'expression de ces croyances. Il revient aux élèves des établissements d'enseignement de se conformer aux règles présidant à une réelle cohésion sociale, à "cette rencontre de tous dans l'espace public». Le port de signes religieux doit être interdit dans les établissements français d'enseignement public. Jean-Paul Willaime constate alors que "dans le contexte français [...] s'agissant de l'articulation entre l'unité politique et la diversité culturelle et religieuse, on promeut plus volontiers un "accommodement raisonné" qu'un "accommodement raisonnable "37 ". Si la loi qui sera adoptée le 15 mars $2004^{38}$ est neutre formellement, elle puise néanmoins sa source dans la recommandation du rapport Stasi. Celui-ci tendait à prohiber le port de signes religieux en général, mais une grande partie de son

$$
+4
$$

36. Ibrd, p. 16

37. Jean-Paul WILLAIME, "Le modèle républicain français d'intégration face à la pluralité relıgieuse contemporaine", Bulletin d'Histoire Poltrqque, vol. 13, n० 3, "La laicité au Québec et en Francen, 2005, p. 63-64.

38. Loi $n^{\circ}$ 2004-228 du 15 mars 2004 encadranc, en application du principe de latcité, le port de signes ou de renues manifestant une appartenance religıeuse dans les écoles, colleges et lycées publics, J.O. (Journal Officiel); $n^{\circ} 65,17$ mars 2004 , p. 5190 . 
argumentaire était consacrée à la signification du foulard islamique en particulier comme contraire au principe fondamental reconnu par les lois de la République qu'est l'égalité entre les hommes et les femmes. Tout en s'inscrivant dans l'idée de séparation des Églises et de l'Érat comme condition de mise en œuvre de la neutralité, cette loi réaffirme l'idéal républicain d'un État porteur d'un magistère philosophique émancipateur d'individus englués dans des appartenances religieuses jugées non compatibles avec les valeurs démocratiques. Seule la séparation permet alors la liberté de penser des citoyen $s^{39}$, et les jeunes femmes musulmanes sont implicitement visées par la loi. L'interdiction du port de signes religieux dans les établissements scolaires publics a donc pu soulever des interrogations quant à la réelle neutralité de l'État à l'endroit de populations qui, de fait, se trouvaient visées et indirectement sanctionnées par les dispositions de cette loi "neutre».

Si ces mises en cause de la réelle neutralité de l'État visent ici des populations minoritaires, force est de constater que des questionnements similaires, mais où la population majoritaire serait cette fois-ci désavantagée, sont apparus récemment au Québec après que la Cour suprême du Canada ait autorisé à un jeune sikh le port du kirpan rituel à l'école.

\section{ON CONNAÎT L'ARBRE, PAS LES FRUITS. LES ACCOMMODEMENTS CANADIENS SOUS TENSION}

Très forte sur le plan symbolique, probablement tout autant qu'a pu l'être la loi du 15 mars 2004 pour la France, une décision de la Cour suprême du Canada rendue le 2 mars 2006 a indiqué la procédure à suivre quant au port de signes religieux dans les écoles canadiennes. Il s'agit de la décision Multani c. Commission scolaire Marguerite-Bourgeoys ${ }^{40}$. La Cour suprême devait alors déterminer si un jeune sikh pouvait porter son kirpan dans l'école publique qu'il fréquentait.

S'appuyant sur une tradition d'interprétation libérale de la Charte des droits, la Cour suprême souligne le droit constitutionnel de liberté de religion du requérant et, après avoir procédé à un examen in concreto des faits, rappelle que le principe de l'accommodement raisonnable doit s'imposer à l'institution scolaire. Ce faisant, tout en assortissant le port de ce

$$
++\div
$$

39. Par "liberté de penser", nous reprenons ici l'idéal type défini par lean Baubérot et selon lequel: "Il ne s'agit plus là d'une conception pluraliste de la liberré, mais de la liberté perçue comme émancipation face à toute doctrine englobante. émancipation effectuée grâce aux unstruments fournis par la raison et la sciencen (Jean BAUbéroT, "Larcité, sectes, société», Françoise Champion et Martine Cohen [dir.], Sectes et démocratie, Paris, Éditıons du Seuil, 1999, p. 316). 40. Multant c. Commoston scolatre Marguerite-Bourgeoys, [2006] 1 R.C.S 256, 2006 CSC 6. 
signe religieux de nombreuses conditions afin qu'aucun trouble à la sécurité ne puisse en résulter, elle refuse de déformer la symbolique religieuse du kirpan en ne le qualifiant pas d'arme potentielle et privilégie le respect de la liberté de religion sur d'hypothétiques atteintes à la sécurité des étudiants. La Cour suprême ne généralise pas pour autant le port du kirpan et sa décision ne fait jurisprudence qu'en ce que la procédure de l'accommodement devient celle que les écoles canadiennes doivent respecter pour décider si des élèves peuvent être individuellement autorisés à porter un signe religieux dans l'enceinte de l'établissement scolaire.

Dans le domaine du port de signes religieux à l'école, la Cour suprême a donc clairement posé la marche à suivre. Et elle indique expressément que cette marche à suivre - l'accommodement raisonnable permet une meilleure prise en "compte des valeurs canadiennes fondées sur le multiculturalisme». Ces valeurs sont aussi essentiellement fondées sur le principe de tolérance qui doit être ici compris comme préalable à l'égalité de tous les citoyens, comme un "dépassement de la simple résignation à la diversité empirique [qui conduit alors] à la reconnaissance de l'altéritéé ${ }^{41}$. En effet, poursuit Micheline Milot,

l'égalité, tant d'un point de vue juridique que social, peut s'exprimer selon des modalités différentes, pourvu que les moyens pour assurer sa mise en œuvre n'affectent pas l'égalité de statut des citoyens, l'égalité des ressources pour la conduite de sa vie et l'égalité des chances ${ }^{42}$,

dont fait partie l'accès à l'éducation.

L'accommodement à la diversité religieuse dans la sphère publique est ici perçu comme une modalité particulière du respect de l'égalité, comme un élément de la politique de reconnaissance de la diversité qui s'inscrit dans les objectifs politiques du multiculturalisme canadien et doit favoriser, à moyen et long terme, l'intégration des minorités dans la société canadienne et ses institutions publiques ${ }^{43}$. Pourtant, "l'affaire Multani" a soulevé de nombreuses controverses. L'accommodement raisonnable est remis en question. La décision de la Cour suprême semble avoir cristallisé dans la population, au Québec en particulier, l'idée qu'il est un privilège indu consenti aux immigrants, accréditant ainsi la position de ceux qui

$$
+4+
$$

41. Michehne MILOT, «La prise en compte des réalités religieuses par les institutions publiques et le respect de la laicité», Viure-Ensemble, vol. 13, $\mathrm{n}^{\mathrm{a}} 47$, printemps-été 2006, p. 4.

42. lbid., p. 4.

43. Pierre BOSSET et Paul EID, "Droit et religıon. De l'accommodement raisonnable à un dialogue internormatif? " htcp://www.cdpdj.qc.ca/fr/publicarions/liste.asp?Sujet=3\&noeud $1=1$ \&noeud $2=68$ cle $=0$ (16 seprembre 2007). 
contestaient la neutralité réelle de cette décision juridique ${ }^{44}$. Micheline Milot constate ainsi que souvent

il n'y a pas vraiment de dialogue, mais une requête non négociable de la part d'ayant-droits, appuyée sur une interprétation procédurale des droits et libertés. Il en résulte [la perception selon laquelle] le croyant [aurait] en quelque sorte un «avantage" sur le non-croyant dans un conflit de normes ${ }^{45}$

et donc celle selon laquelle l'État ne serait pas pleinement neutre dans la gestion de ce conflit. L'effer symbolique, sur le plan des relations sociales, n'est pas "négligeable et semble nourrir ainsi une méfiance réciproque ou un sentiment d'iniquité [et] la valorisation de la diversité pourrait alors être perçue comme un obstacle à l'intégration sociale ${ }^{46}$ ". Au Québec, la polémique relative aux accommodements religieux reste d'ailleurs particulièrement d'actualité.

Les débats portant sur le port de signes religieux à l'école concernent la question fondamentale de l'intégration des religions minoritaires dans l'espace public. Depuis 2004, les politiques adoptées par la France et le Canada divergent radicalement dans le choix des modalités de cette intégration. En adoptant la loi du 15 mars 2004, la France a réaffirmé son attachement à un modèle où la séparation des Églises et de l'État est envisagée comme un préalable, une condition de mise en ouvre de la neutralité dans l'institution publique qu'est l'école. À l'inverse, la Cour suprême du Canada, en imposant des accommodements raisonnables aux institutions scolaires, promeut une séparation plus souple, envisagée comme "composante effective de la neutralité" de l'État.

En 2003, le rapport Stasi indiquait que si l'administration devait donner toutes les garanties de la neutralité, elle devait aussi en présenter les apparences afin que l'usager ne puisse douter de sa réelle neutralité. En France et au Québec, les politiques adoptées quant au port de signes religieux dans les écoles ont été fortement critiquées et l'effective neutralité des États

$$
++
$$

44. Pierre Bosset relève notamment que " par crainte de dérives ou compromissions, notamment face à la revendication milıtante de parricularismes religieux, la légitimıté de l'obligation de l'accommodement raisonnable comme outil de régulation de la diversité relıgieuse se trouve parfois remıse en question au Québec. Pour plusıurs, l'approche de "laucité ferme" incarnée dans la loi française du 15 mars 2004 apparaîtra comme une solution de rechange" (Pierre BOSSET, "Le droit et la régulacion de la diversıté religıeuse en France et au Québec. Une même problématıque, deux approches", Bulletzn d'Histoire Politique, vol. 13, $n^{\circ}$ 3, "La laicité au Québec et en France», 2005, p. 90).

45. Micheline MiLOT, "La prise en compte des réalırés religieuses par les institutions publiques er le respecr de la laicité ", p. 6.

46. Ibid. 
français et canadien, largement remise en question. D'une part, elles ont été perçues comme une nouvelle stigmatisation d'une communauté musulmane en mal d'intégration. D'autre part, elles ont été reçues comme une concession supplémentaire accordée à des minorités systématisant leurs revendications. Au Québec en particulier, l'accommodement consenti au jeune sikh par la Cour suprême a donné lieu à un tollé et à une véritable " tempête politique ${ }^{47}$ " laissant croire à la majorité qu'on la dépouillait de son identité. Quel que soit le mode de régulation du religieux choisi, les sentiments de partialité ont donc surgi de part et d'autre. Malgré les inquiétudes des populations et leurs attentes de solutions immédiates quant à leurs questionnements identitaires, ces politiques de régulation du religieux ne peuvent être évaluées à court terme. Elles sont aussi un pari sur l'avenir. Mais à court terme, ces perceptions de partialité de l'État dans le traitement du port de signes religieux dans les écoles limitent la portée, en matière de neutralité, des politiques publiques qui ont été adoptées.

47. Daniel WEINSTOCK, "La "crise" des accommodements au Quebec. Hyporhèses explıcatives", Ethique publique, vol. 9, $\mathrm{n}^{\circ} 1$, "L'aménagement de la diversiré culturelle ec religieusen, 2007, p. 20. 